\title{
Evolution de la fréquence du cornage dans quatre races caprines françaises
}

\author{
G. RICORDEAU, F. SANCHEZ Y G.F. (*) \\ avec la collaboration de A. Galllard \\ I.N.R.A., Station d'Amélioration génétique des Animaux, C.R. de Toulouse \\ B.P. 12, F-31320 Castanet-Tolosan
}

\begin{abstract}
Résumé
L'évolution de la fréquence du gène avec cornes $(p)$ a été étudiée de 1967 à 1979 dans quatre populations caprines françaises grâce aux déclarations de naissance (D.N.) des animaux conservés pour la reproduction.

Pour les mâles et femelles nés en 1979 , cette fréquence $\left(q_{p}\right)$ est maximum en race Chamoisée $(0,88$ et 0,89 respectivement), minimum dans les races Saanen $(0,77$ et 0,78$)$ et Poitevine $(0,81$ et 0,76$)$, intermédiaire en race Croisée $(0,82$ et 0,83$)$. Le pourcentage de produits nés de deux parents sans cornes est de 4 à 5 p. 100 en Chamoisée contre 14 à 31 p. 100 dans les trois autres races. Dans l'ensemble, la fréquence du cornage des produits est supérieure à celle de leur mère, mais comparable à celle de leur père.

En race Chamoisée, on peut espérer obtenir plus de 95 p. 100 de chèvres cornues en quatre générations dans l'hypothèse d'une utilisation exclusive de boucs cornus. Le progrès sera plus lent en race Saanen, dans la mesure où les éleveurs sélectionnent aussi les homozygotes blancs, et surtout en race Poitevine, compte tenu des faibles effectifs et des impératifs du programme de conservation.
\end{abstract}

\section{Introduction}

Jusqu'en 1970, à l'exemple des Suisses, les éleveurs caprins français ont sélectionné en faveur du gène sans cornes $P$, autosomal dominant son allèle $p$ pour le cornage normal. A partir de cette période, les travaux originaux de l'I.N.R.A., ainsi que les recherches effectuées à l'étranger, ont clairement montré que toutes les femelles homozygotes sans cornes $P P$ étaient masculinisées en intersexués et pseudomâles - donc impropres à la reproduction - et que 91 p. 100 des mâles $P P$ étaient totalement ou partiellement stériles, de sorte que la sélection d'une lignée pure sans cornes est non seulement impossible mais d'approche coûteuse (cf. RicordeAu \& Lauvergne, 1967 ; Weber, 1969 ; ainsi que les revues de Boyajean, 1969 et LAUVERgNe, 1969). Aussi les objectifs de la sélection ont-ils été progressivement

(*) Universitad Autonoma Metropolitana, Xochimilco - Mexico. 
modifiés. Compte tenu de son importance, le caractère cornage a été pris en compte dans les enregistrements du contrôle de performances, sur les documents de saillies, d'insémination artificielle, de mise-bas et les déclarations de naissance (D.N.). Ceci permet d'éviter les accouplements entre parents sans cornes, de vérifier les paternités et d'encourager les éleveurs à retenir des boucs cornus. Par ailleurs, depuis 1970, les trois centres de testage français (S.E.I.A., 86480 Rouillé ; Midacaprin, 81110 Soual ; Capricentra, 86600 Mignaloux) retiennent en priorité des boucs cornus et refusent les boucs sans cornes issus de deux parents mottes.

La présente étude a pour but d'analyser l'évolution de la fréquence du gène cornage chez les reproducteurs des deux sexes dans l'ensemble de la population caprine améliorée française, pour estimer la façon dont la sélection en faveur des animaux cornus a réellement été pratiquée.

\section{Matériel et méthodes}

Nous avons utilisé dans notre étude les déclarations de naissance (D.N.) des reproducteurs inscrits au "fichier racial» de l'U.P.R.A. Elles concernent les animaux nés de 1967 à 1976 et en 1979 (les D.N. des animaux nés en 1977 et 1978 n'ont pas été retenues afin d'éliminer totalement certaines erreurs introduites lors du changement de gestion du fichier en 1977). Il s'agit de 8644 D.N. de mâles et 76326 D.N. de femelles, appartenant à 4 types génétiques : Alpin chamoisé, Saanen, Poitevin et Croisé (tous les reproducteurs qui n'appartiennent pas aux 3 autres races). La Poitevine, classée comme race à faible effectif, fait l'objet d'un «programme de conservation » avec planification des accouplements, rotation des mâles et conservation des chèvres aussi longtemps que possible, afin de limiter le niveau de consanguinité (Malafosse, 1977 ; Chevalet \& de Rochambeau, 1979). L'année de naissance $n$ va du $1^{\text {er }}$ octobre de l'année n-1 au 30 septembre de l'année n.

Le taux d'incompatibilité des D.N. a été estimé d'après le pourcentage de produits sans cornes issus de deux parents cornus.

La fréquence phénotypique du caractère avec cornes étant égale au rapport de l'effectif d'animaux cornus $(n)$ à l'effectif total $(N)$, la fréquence génique $\left(q_{p}\right)$ est $\sqrt{\frac{\mathrm{n}}{\mathrm{N}}}$. La pression de sélection en faveur des animaux cornus est estimée d'après l'importance des accouplements entre parents sans cornes et la comparaison des pourcentages observés et théoriques de produits cornus en fonction du cornage des parents.

\section{Résultats}

\section{A. - Taux d'incompatibilité}

Nous vérifions que le taux d'incompatibilité est toujours inférieur à 5 p. 100 (tabl. 1). 


\section{TABLeau 1}

Taux d'incompatibilité des déclarations de naissance (D.N.) : pourcentage de produits sans cornes issus de deux parents cornus

Incompatibility rate of birth declaration (D.N.) : percentage of hornless offsprings born from two horned parents

\begin{tabular}{|c|c|c|}
\hline Races & Mâles & Femelles \\
\hline Chamoisée & $4,2(1528)$ & $3,0(12457)$ \\
\hline Saanen ... & $0,9 \quad(223)$ & $3,2 \quad(1759)$ \\
\hline Poitevine .. & (3) & (34) \\
\hline Croisée .................... & $4,7 \quad(107)$ & $4,4 \quad(1509)$ \\
\hline
\end{tabular}

B. - Fréquence du phénotype cornu et du gène $\mathrm{p}$, par année de naissance

En race Chamoisée, de 1970 à 1979, la proportion de cornus passe de 71 à 78 p. 100 chez les mâles, de 30 à 79 p. 100 chez les femelles. En race Saanen, ce pourcentage passe pour la même période de 33 à 60 p. 100 dans les deux sexes, si l'on considère que l'échantillon des chevrettes nées en 1970 est vraisemblablement peu représentatif. En race Poitevine, la tendance est moins régulière, compte tenu des faibles effectifs. Chez les Croisés, les pourcentages sont intermédiaires à ceux observés en Saanen et Chamoisée (tabl. 2, fig. 1).

\section{TABLEAU 2}

Proportions de cornus chez les fils et les filles et chez leurs parents, suivant l'année de naissance du produit

Horned phenotypic frequency in sons and daughters, and their parents, according to the birth-year of the offspring

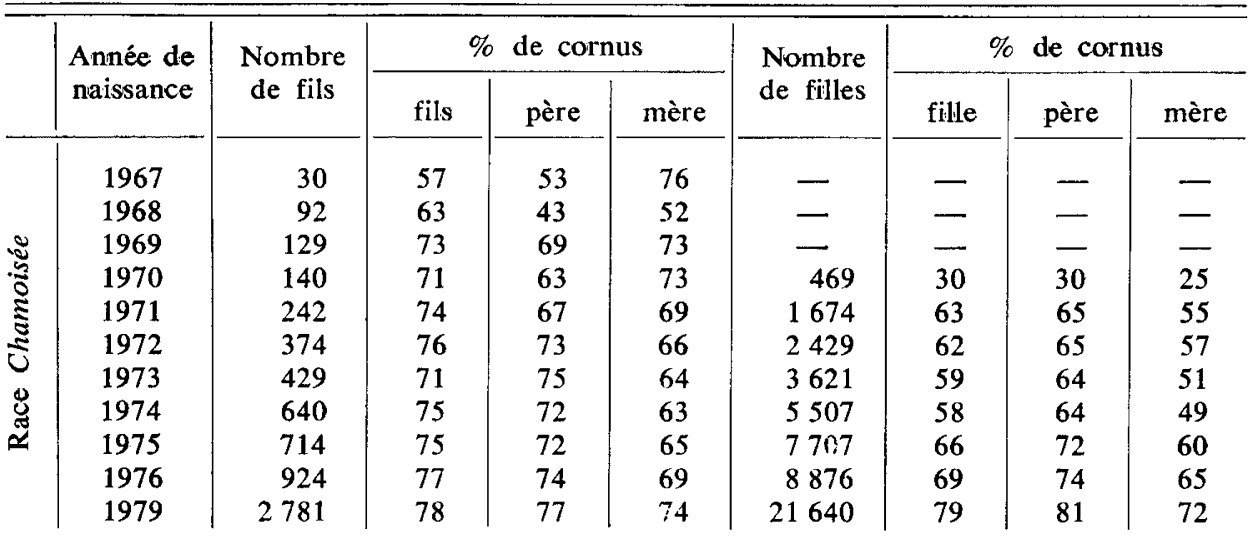


TABLEAU 2 (suite)

\begin{tabular}{|c|c|c|c|c|c|c|c|c|c|}
\hline & \multirow{2}{*}{$\begin{array}{l}\text { Année de } \\
\text { naissance }\end{array}$} & \multirow{2}{*}{$\begin{array}{l}\text { Nombre } \\
\text { de fils }\end{array}$} & \multicolumn{3}{|c|}{$\%$ de cornus } & \multirow{2}{*}{$\begin{array}{l}\text { Nombre } \\
\text { de filles }\end{array}$} & \multicolumn{3}{|c|}{$\%$ de cornus } \\
\hline & & & fils & père & mère & & fils & père & mère \\
\hline \multirow{9}{*}{ 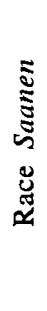 } & 1969 & 13 & 31 & 8 & 21 & - & - & - & - \\
\hline & 1970 & 30 & 33 & 30 & 10 & 47 & 2 & 2 & 0 \\
\hline & 1971 & 30 & 33 & 17 & 45 & 176 & 32 & 29 & 23 \\
\hline & 1972 & 75 & 39 & 17 & 25 & 398 & 31 & 29 & 26 \\
\hline & 1973 & 90 & 40 & 29 & 30 & 666 & 28 & 24 & 32 \\
\hline & 1974 & 135 & 43 & 33 & 34 & 1200 & 37 & 32 & 31 \\
\hline & 1975 & 162 & 51 & 44 & 36 & 1738 & 43 & 35 & 41 \\
\hline & 1976 & 235 & 53 & 40 & 36 & 1733 & 51 & 51 & 47 \\
\hline & 1979 & 891 & 60 & 62 & 44 & 5973 & 60 & 61 & 50 \\
\hline \multirow{8}{*}{ 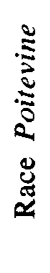 } & 1970 & 5 & 20 & 0 & 40 & 15 & 0 & 0 & 0 \\
\hline & 1971 & 13 & 38 & 8 & 38 & 100 & 48 & 26 & 30 \\
\hline & 1972 & 10 & 30 & 30 & 30 & 98 & 38 & 23 & 31 \\
\hline & 1973 & 15 & 33 & 7 & 47 & 126 & 40 & 20 & 63 \\
\hline & 1974 & 9 & 55 & 56 & 78 & 120 & 46 & 34 & 57 \\
\hline & 1975 & 8 & 50 & 75 & 25 & 127 & 51 & 39 & 53 \\
\hline & 1976 & 12 & 67 & 33 & 38 & 108 & 49 & 52 & 27 \\
\hline & 1979 & 20 & 65 & 65 & 25 & 182 & 57 & 35 & 52 \\
\hline \multirow{8}{*}{ 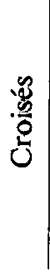 } & 1970 & 4 & - & - & - & 1127 & 30 & 2 & 2 \\
\hline & 1971 & 6 & 67 & 33 & 83 & 1443 & 34 & 9 & 10 \\
\hline & 1972 & 9 & 44 & 66 & 33 & 1234 & 45 & 15 & 17 \\
\hline & 1973 & 18 & 56 & 44 & 66 & 666 & 52 & 45 & 38 \\
\hline & 1974 & 10 & 50 & 50 & 60 & 746 & 55 & 51 & 49 \\
\hline & 1975 & 22 & 68 & 54 & 41 & 1096 & 62 & 60 & 54 \\
\hline & 1976 & 12 & 50 & 42 & 42 & 1326 & 60 & 62 & 55 \\
\hline & 1979 & 315 & 68 & 60 & 56 & 3958 & 69 & 67 & 56 \\
\hline
\end{tabular}

Tableau 3

Fréquence du gène avec cornes $\left(q_{p}\right)$ chez les produits nés en 1979

Gene frequency of horned gene $\left(q_{p}\right)$ in the offsprings born in 1979

\begin{tabular}{|c|c|c|}
\hline Races & Mâle & Femelle \\
\hline Chamoisée ... & 0,882 & 0,890 \\
\hline Saanen & 0,775 & 0,778 \\
\hline Poitevine & 0,806 & 0,756 \\
\hline Croisée & 0,822 & 0,829 \\
\hline
\end{tabular}



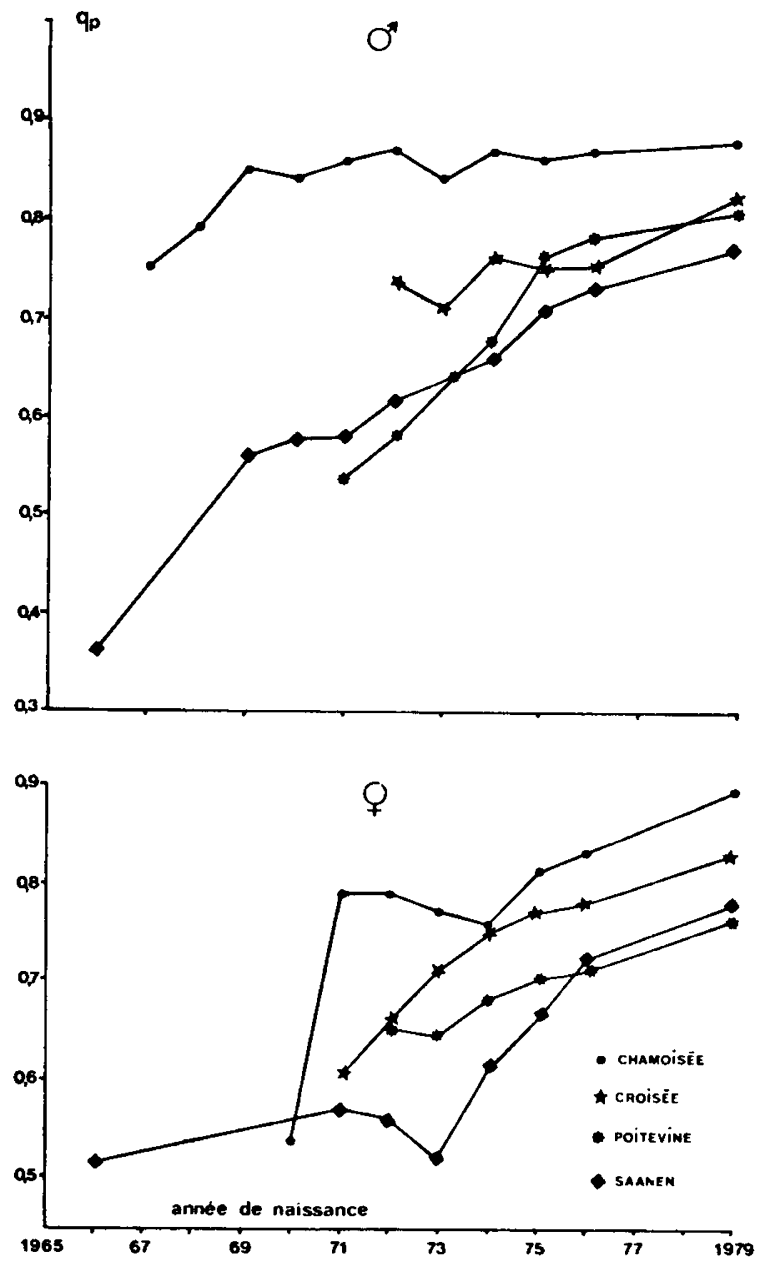

FIG. 1

Evolution de la fréquence du gène sans cornes p pour les quatre races de 1966 à 1979 (mâles et femelles)

Evolution of the horned gene frequency $\left(q_{p}\right)$ in four breeds, from 1966 to 1979

Note : Pour la race Poitevine et les Croisées, nous avons calculé la moyenne mobile afin de limiter les variations annuelles.

Nous pouvons exprimer ces résultats en termes de fréquence génique $\left(q_{p}\right)$ pour les animaux nés en 1979 (tabl. 3) : cette fréquence est maximum en race Chamoisée $(0,88$ et 0,89 pour les mâles et femelles), minimum dans les races Saanen $(0,77$ et 0,78$)$ et Poitevine $(0,80$ et 0,76$)$ et intermédiaire chez les Croisés $(0,82$ et 0,83$)$. 


\section{TABleau 4}

Sélection en faveur des produits cornus suivant le type d'accouplement: D.N. des produits nés en 1979

Selection in favour of the horned offsprings (D.N. 1979)

a) Importance des accouplements entre parents sans cornes (en p. 100) Matings with hornless parent (p. 100)

\begin{tabular}{|c|c|c|}
\hline Race & Mâles & Femelles \\
\hline Chamoisée & $4,7(2781)$ & $4,2(21.640)$ \\
\hline Saanen & $18,6 \quad(891)$ & $17,9 \quad(5973)$ \\
\hline Poitevine & 25,0 & 31,3 \\
\hline Croisée ........... & $18,4 \quad(315)$ & $14,8 \quad(3958)$ \\
\hline
\end{tabular}

b) Pourcentage de produits cornus suivant le type d'accouplement Percentage of horned offsprings according to the type of mating

\begin{tabular}{|c|c|c|c|c|}
\hline \multirow{2}{*}{ Race } & \multicolumn{2}{|c|}{2 parents sans cornes } & \multicolumn{2}{|c|}{$\begin{array}{c}1 \text { parent cornu } \times 1 \text { parent } \\
\text { sans cornes }\end{array}$} \\
\hline & Mâles & Femelles & Mâles & Femelles \\
\hline Chamoisée & $34,6(130)^{* *}$ & $40,0 \quad(915)^{* *}$ & $58,5\left(\begin{array}{lll}1 & 123\end{array}\right)^{* *}$ & $56,7(8268)^{* *}$ \\
\hline Saanen .. & $34,9(166)^{* *}$ & $34,6(1071)^{* *}$ & $51,0 \quad(502) \mathrm{NS}$ & 49,1 (3 143) NS \\
\hline Poitevine & (5) NS & (57) NS & (12) NS & (91) NS \\
\hline Croisée. & $27,6 \quad(58) \cdot \mathrm{NS}$ & $(585)^{* *}$ & $(150)^{* *}$ & $54,4(1864)^{* *}$ \\
\hline p. 100 théorique & \multicolumn{2}{|c|}{25} & \multicolumn{2}{|c|}{50} \\
\hline
\end{tabular}

Signification des écarts par rapport aux pourcentages théoriques : très significatif $(* *)$ ou non significatif (NS).

c) Influence de la période de naissance Influence of the birth period

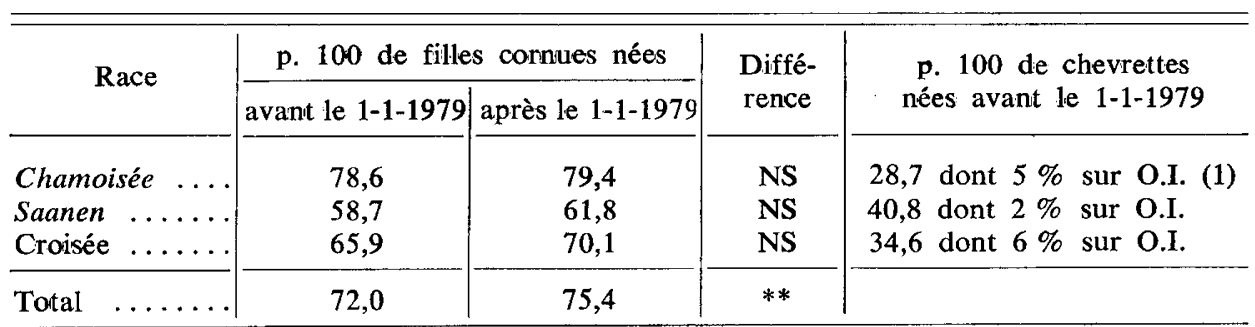

(1) O.I. : Oestrus Induit. 


\section{C. - Pression de sélection en faveur du caractère cornu}

Elle a été estimée pour les animaux nés en 1979 dans le tableau 4.

Les produits mâles et femelles nés de deux parents sans cornes représentent 4 à 5 p. 100 des cas en Chamoisée contre 14 et 19 p. 100 en Croisée, 17 et 19 p. 100 en Saanen, 25 et 31 p. 100 en Poitevine (tabl. 4 a).

Dans l'ensemble, sauf en Poitevine où les effectifs sont faibles, la proportion de cornus chez les produits (fils ou fille) est comparable à celle de leur père, mais nettement supérieure à celle de leur mère (tabl. 2).

Par ailleurs, si l'on compare les pourcentages observés et théoriques de produits cornus nés des accouplements entre deux parents sans cornes, ou entre deux parents dont l'un est cornu, les écarts sont toujours significatifs en race Chamoisée, significatifs dans 3 cas sur 4 en Croisée et uniquement significatifs dans le cas des produits issus de parents sans cornes en Saanen (tabl. 4 b). On remarque également que, lors du choix des chevrettes sans cornes, l'éleveur tient probablement compte de leur date de naissance, puisque le pourcentage de filles cornues est toujours moins élevé parmi celles nées précocement (d'octobre à décembre), mais la différence n’est pas significative intra race (tabl. $4 \mathrm{c}$ ).

\section{Discussion}

\section{A. - En race Chamoisée}

Dans l'ensemble, l'effort de sélection pour augmenter la proportion d'animaux cornus a été plus important en race Chamoisée. La fréquence génique est passée de 0,45 en 1961-1962 (estimation sur 450 chèvres RICORDEAU, 1963) à 0,89 en 1979. Par ailleurs, dans cette race, depuis 1977, plus de 90 p. 100 des boucs réservés au testage en station sont cornus. Compte tenu de la fréquence génique actuelle et en supposant - hypothèse réaliste - l'utilisation exclusive de mâles cornus, on peut donc espérer atteindre la fréquence de 0,99 (soit 98 p. 100 d'animaux cornus) en quatre générations, c'est-à-dire, d'ici 15 ans (l'intervalle moyen de génération étant de 3,6 années : RicORDEAU et al., 1979). On pourrait chercher à maintenir un minimum de reproducteurs sans cornes, pour continuer à produire des chèvres hétérozygotes $P p$ qui présentent un avantage sélectif de l'ordre de 6 p. 100 au niveau de la prolificité (Soller \& Kempenich ; Ricordeau, 1969 ; Hancock \& Louca, 1975) ou de l'effet maternel (RICORDEAU et al., 1972). Cependant, cette solution n'est pas souhaitable, car elle conduirait également à produire des boucs hétérozygotes qui seraient exclus de la reproduction.

\section{B. - En race Saanen}

Dans cette race la fréquence génique est passée de 0,50 à partir de $1961(0,48$ sur 805 chèvres de la Drôme en 1961 : Rrcordeau, 1963 ; 0,53 sur les chèvres de Lozère en 1965-1967 : RICORDEAU \& LAUVERGNE, 1967) à 0,77 en 1979. Dans cette 
race, il est difficile de définir une situation de sélection sur le cornage, dans la mesure où les éleveurs cherchent également à sélectionner des reproducteurs homozygotes blancs (le gène Rouan est dominant : Ricordeau \& Lauvergne, 1971). Cependant, dès maintenant, il faut insister sur le nombre de boucs issus de deux parents sans cornes : 166 sur 891 en 1979, dont 108 (soit 12,1 p. 100) sans cornes hétérozygotes ou homozygotes en majorité stériles (tableau 4 a). C'est là un risque non négligeable qui doit affecter la fertilité de certains troupeaux et qu'il faudrait limiter impérativement en maintenant au moins un bouc cornu dans chaque élevage ou en faisant appel à l'insémination artificielle.

\section{C. - En race Poitevine}

En race Poitevine, le problème est loin d'être résolu, puisque le programme des «accouplements imposés» pour maintenir la variabilité génétique laisse peu de possibilités à une sélection en faveur des animaux cornus.

\section{Conclusion}

Dans l'ensemble de la population caprine française, la nécessaire augmentation de la proportion d'animaux cornus - qui devrait permettre de concentrer les efforts de sélection sur les caractères laitiers - est lente. Pendant trop longtemps, en effet, la sélection contre le cornage a été un obstacle à la sélection pour la production de lait. En Suisse, par exemple, GaIllard \& KROPF (1978) estiment qu'avec une race cornue, la réponse à la sélection sur les performances laitières peut être supérieure de 40 p. 100 à celle obtenue avec une race sans cornes. Il s'agit là d'un résultat extrême, peut-être, mais à garder en tête.

Reçu pour publication en octobre 1981.

\section{Remerciements}

Nous tenons à remercier C. DU SARTEL, directeur de l'U.P.R.A. caprine, qui nous a communiqué la première partie du fichier racial, ainsi que le $\mathbf{P}^{r} \mathrm{D}^{r} \mathrm{~W}$. WeBER - Berne, Suisse - et J.J. Lauvergne, qui ont bien voulu lire et corriger ce texte.

\section{Summary \\ Horned gene frequencies in four French goat breeds}

The frequencies of the gene for horned character has been estimated from 1967 to 1979 among the 4 French goat breeds according to the birth declarations of the animals selected for reproduction (8644 males and 76326 females).

As for the males and females born in 1979, this frequency is maximum in Chamois breed (0.88 and 0.89 resp.), minimum is Saanen $(0.77$ and 0.78$)$ and Poitevine $(0.81$ and 0.76$)$, intermediate in the Crossbred $(0.82$ and 0.83$)$. 
The percentage of youngs born from two polledness parents amounts to 4 to 5 p. 100 for the Chamois, versus 14 to 31 p. 100 for the three other breeds. As a whole, horned gene frequencies of the products are more than their mother's one but similar to their father's one.

In Chamois breed, over 95 p. 100 horned goats could be obtained within four generations, in the hypothesis where only horned males would be used. The gain will be slowler in Saanen, given that breeders also hope to select homozygotes for white colour, and mostly in Poitevine, given the low number of animals and the requirements of the conservation schemes.

\section{Références bibliographiques}

Boyajean D., 1969. Intersexualité associée à l'absence de cornes chez la chèvre Alpine. Ann. Génét. Sél. anim., 1, 447-463.

Chevalet C., Rochambeau H. de, 1979. Gestion des petites populations. V ${ }^{\text {es }}$ Journées de la Recherche ovine et caprine. I.N.R.A.-I.T.O.V.I.C., Paris, 282-292.

Gaillard C., KropF W., 1978. Theoretische und praktische Möglichkeiten der genetischen Verbesserung der Ziegenbestände. Schr. Schweiz. Ver. Tierzü̈cht, 54, 20-31.

Hancock J., Louca A., 1975. Polledness and intersexuality in the Damascus breed of goat. Anim. Prod., 21, 227-231.

Lauvergne J.J., 1969. Progrès des connaissances génétiques sur l'intersexualité associée à l'absence de cornes chez la chèvre d'origine Alpine. Ann. Génét. Sél. anim., 1, 403-412.

LAUVERGNe J.J., 1969. Fréquences à l'équilibre du gène d'origine alpine responsable de l'absence de cornes et de l'intersexualité dans des populations de chèvres. Ann. Génét. Sél. anim., 1, 413-422.

Malafosse A., 1977. Le programme Poitevin. Polycopié, U.P.R.A., I.N.R.A., I.T.O.V.X.C., $30 \mathrm{p}$.

Ricordenu G., 1963. Possibilités de sélection dans l'espèce caprine. Bull. tech. Inf. Ing. Serv. Agric. (179), 189-204.

Ricordeau G., 1969. Surprolificité des génotypes sans cornes dans les races Alpine Saanen, Alpine Chamoisée et Poitevine. Ann. Génét. Sél. anim., 1, 391-395.

Ricordeau G., LaUvergne J.J., 1967. Hypothèse génétique unique pour expliquer la présence d'intersexués, de mâles en excès et de mâles stériles en race caprine Saanen. Ann. Zootech., 16, 323-334.

Ricordeau G., Lauvergne J.J., 1971. Déterminisme héréditaire de la couleur blanche de la chèvre Saanen. Ann. Génét. Sél. anim., 3, 425-432.

Ricordeau G., Poujardieu B., Bouillon J., 1972. Effet maternel du gène sans cornes $P$ sur le poids des chevrettes d'élevage. Ann. Génét. Sél. anim., 4, 29-34.

Ricordeau G., Bouillon J., Sanchez F., Mocquot J.C., Lajous A., 1979. Amélioration génétique des caprins. Facteurs favorisant ou limitant le progrès génétique. $\mathrm{V}^{\mathrm{cs}}$ Journées de la Recherche ovine et caprine. I.N.R.A.-I.T.O.V.I.C., Paris, 403-426.

Soller M., Kempenich O., 1964. Polledness and litter size in Saanen goats. J. Hered., 55, 301-304.

Weber W., 1969. La stérilité des mâles dans les races caprines Suisses sans cornes. Ann. Génét. Sél. anim., 1, 379-382. 